\title{
$\mathrm{Li} / \mathrm{Li}(100)$ 体系的吸附与表面 扩散的对势研究
}

\author{
王泽新夏少武王建新
}

(骨出化工学院应用化学系, 青舟 266042)

关链词对势和方法研究、吸附和表面扩散、 $\mathrm{Li} / \mathrm{Li}(100)$ 体系的研究

原子、分子同固体表面的相互作用是多相催化, 气体腐蚀, 分离以及晶体生长等领域研究 中的一个重要课题. 从分子水平上进行理论研究, 不仅有助于认识吸附质和表面相互作用机 理, 而且可以获得吸附、表面扩散等动力学信息. 尤其是台阶缺陷表面对吸附、扩散扰动的研 究,使我们对气固吸附本质有进一步的认识.

处理吸附质和固体表面相互作用的方法有刚性能带模型 ${ }^{[1,}$, 相互作用分子近似 ${ }^{[2]}$. 比较常 用的方法是准分子模型, 选取有限数量的原子构成 Cluster 模拟固体表面, 同吸附分子组成一 个巨分子来处理 ${ }^{[3]}$. 该方法对于吸附作用可以给出直观、定域的图象. 而用 $a b$ initio 打 法 及半经验的 CNDO、EHMO、DIM 等方法处理 Cluster 时, 不能选用足够数目的粒子, 比较 完整地描述平坦的固体表面和台阶缺陷表面, 因此很难深人地研究吸附和表面扩散问题.

选取对势和来描述多体粒子相互作用是常用的一种方法. 胶体粒子的稳定性理论一 DLVD 理论, 就是该法的杰作, 近年来, 开始用于处理气一固表面相互作用 ${ }^{[4]}$. 本文拟采用对势 和法, 并引人实验参数来描述吸附质和表面相互作用. 其优点是可选取足够大的 Cluster 来 模拟定态的理想平坦表面和台阶缺陷表面, 给出吸附质和表面相互作用的定域描述, 并能充 分体现出晶格的平移对称性.

\section{一、方法和模 型}

若将被吸附原子与固体表面的相互作用势 $U(\boldsymbol{r})$ 看作该原子与表面原子之间的相互作 用势 $V_{i}(\boldsymbol{r})$ 之和, 则

$$
U(\boldsymbol{r})=\sum_{i} V_{i}(\boldsymbol{r}),
$$

其中 $i$ 遍及 Cluster 诸原子. $V_{i}(\boldsymbol{r})$ 可用 Morse 势表示为

$$
V_{i}(\boldsymbol{r})=D\left[e^{\left.-2 \alpha\left(\left|\boldsymbol{r}-\boldsymbol{r}_{i}\right|\right)-r_{0}\right)}-2 e^{-\alpha\left(\left|\boldsymbol{r}-\boldsymbol{r}_{i}\right|-\boldsymbol{r}_{0}\right)}\right],
$$

式中 $D 、 \alpha$ 和 $r_{0}$ 可作为模拟参数处理.

金属 $\mathrm{Li}$ 属 bcc 晶格, $\mathrm{Li}(100)$ 面是常研究的解理面. 我们采用长 $\times$ 宽 $\times$ 高为 $13 \times 13 \times 7$ 层晶胞原子模找理想 $\mathrm{Li}(100)$ 平坦表面, 共包含 2046 个 $\mathrm{Li}$ 原子, 这样使等价吸附位的结合 能误差小于 $10^{-9} \mathrm{eV}$, 同时可消除所研究区域的边界效应. 通过优选模拟参数 (表 1 ), 得到 $\mathrm{Li}$ 原子在 $\mathrm{Li}(100)$ 面上顶位 $(\mathrm{T})$ ）桥位 $(B)$ 和洞位 $(H)$ 的吸附特性 (见表 2 ), 同实验结果相符合.

* 国家自然科学基金资助项目. 
表 1 模拟参数 $D 、 \alpha 、 r_{0}$ 的优选结果

\begin{tabular}{c|c|c}
\hline$D(\mathrm{eV})$ & $\alpha\left(\AA^{-1}\right)$ & $r_{0}(\AA)$ \\
\hline 0.139175 & 0.9079 & 3.5 \\
\hline
\end{tabular}

表 2 Li 在 $\mathrm{Li}(100)$ 平坦表面的吸附特性

\begin{tabular}{|c|c|c|c|c|}
\hline 吸附位 特性 & 结合能/(eV) & 吸附高度 $/(\AA)$ & 局部对称性 & 郎分实验结果 \\
\hline 顶位 ( $\mathrm{T}$ ) & 1.03 & 2.70 & $c_{*}$ & 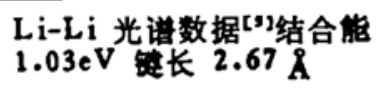 \\
\hline 桥位 (B) & 1.24 & 2.40 & $c_{20}$ & \\
\hline 洞位 (H) & 1.54 & 1.75 & $c_{4,}$ & 晶格常数的 $1 / 2$ \\
\hline
\end{tabular}

为了模拟台阶缺陷表面, 需在平坦表面上铺设部分原子, 通常用 $m\left(h^{\prime} k^{\prime} l^{\prime}\right) \times n\left(h^{\prime \prime} k^{\prime \prime} l^{\prime \prime}\right)-$ [ $u v w]$ 符号表示台阶面, 其中上台面 $\left(h^{\prime} k^{\prime} l^{\prime}\right)$ 和台阶面 $\left(h^{\prime \prime} k^{\prime \prime} l^{\prime \prime}\right)$ 分别由 $m$ 和 $n$ 个 $[u v w]$

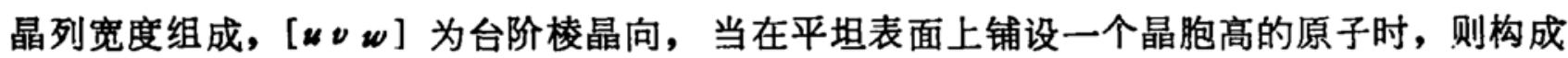
$12(100) \times 2(010)-[001]$ 的台阶缺陷表面.

我们考查了 $\mathrm{Li}$ 在平坦表面和台阶表面的吸附扩散行为,整个计算采取刚性表面近似。

\section{二、结果和讨论}

图 1 给出了 $\mathrm{Li}$ 在 $\mathrm{Li}(100)$ 平坦表面的吸附, 扩散势能面, 其中图 1(1)、(2) 分别为 $\mathrm{Li}$ 原子从顶位 ( $\mathrm{T}$ ) 向桥位 (B) 和从桥位 (B) 向洞位 $(\mathrm{H})$ 的吸附扩散行为, $\mathrm{Z}$ 为 $\mathrm{Li}$ 原子距表面 的高度. 由图可见, 洞位为稳定的谷点吸附位, 顶位和桥位分别为亚稳的峰点和鞍点吸附位. $\mathrm{Li}$ 原子沿表面最低能量扩散通道 $\mathrm{H} \rightarrow \mathrm{B} \rightarrow \mathrm{H}$ 方向, 扩散活化能 $E_{\mathrm{HB}}^{0}=0.30 \mathrm{eV}$. 图 1(3)、 (4)给出 $\mathrm{Li}$ 原子在距表面不同高度并和表面平行的扩散行为, 平面内包含三种不等价的吸附 位. 从图 1 看出, 随着吸附原子愈远离表面, 表面扩散势的变化愈趋于平缓, 体现了远表面区 和近表面区的扩散势特性,它同精确的 ab initio 的分析结果一致 ${ }^{[3]}$.

图 2 给出了在 $12(100) \times 2(010)-[001]$ 台阶表面, $\mathrm{Li}$ 沿能量最低通道分别由上、下台 面向台阶面扩散的吸附、扩散势能面. 高度 $Z$ 的零点取在下台面, 横坐标的零点取在台阶的位 置, 单位为晶格常数 $a$. 表 3 和表 4 集中了吸附、扩散特征结果. 由图 2(1) 明显看到随着 $\mathrm{Li}$ 沿上台面向台阶面扩散:（1)吸附位的结合能逐渐降低, 在紧靠台阶的洞位与 $\mathrm{H}_{2}$ 的结合能 为 $1.33 \mathrm{eV}$, 同平面洞位的结合能 $1.54 \mathrm{eV}$ 比较, 结合能减少 $0.21 \mathrm{eV}$, 吸附几何也有微小变化; (2)扩散活化能逐渐增高, 从 $\mathrm{H}_{2}$ 位向下台面的扩散活化能约为平面扩散活化能 $\boldsymbol{E}_{\mathrm{H} \mathbf{B}}^{*}$ 的 2 倍, 由 $\mathrm{H}_{2}$ 向 $\mathrm{H}_{1}$ 位扩散活化能约为 $\boldsymbol{E}_{\mathrm{HB}}^{\boldsymbol{a}}$ 的 1/2. 在图 2(2) 可见, 在下台面情况正相反, 随着 $\mathrm{Li}$ 原子向台阶位的扩散: (1) 吸附位结合能逐渐增高, 在靠近台阶的洞位 $\mathrm{H}_{3}$, 其结合能比平面 洞位结合能高 $0.64 \mathrm{eV}$; (2) 扩散活化能逐步降低, 从 $\mathrm{H}_{4}$ 向 $\mathrm{H}_{3}$ 的扩散活 化能约为 $E_{\mathrm{HB}}^{*}$ 的 $1 / 5$, 而从 $\mathrm{H}_{3}$ 向 $\mathrm{H}_{4}$ 的远离台阶位的扩散活化能约为 $E_{\mathrm{HB}}^{\mathrm{a}}$ 的 2 倍, 向上台面的扩散活化能约 为 $E_{\mathrm{HB}}^{a}$ 的 5 倍. 由此可见, 台阶的扰动形成了粒子的异常吸附扩散势. 即粒子沿上台面向 台阶处扩散, 需要克服愈来愈高的势垒, 相当于爬坡, 而远离台阶的扩散比较容易, 在台阶处形 成反射势. 粒子沿下台面向台阶处扩散, 需要克服的势垒愈来愈低, 相当于下坡, 在台阶处形 成捕获势. 上述结果同精确的 $a b$ initio 结果一致 ${ }^{[3]}$, 并从理论上支持了 Ehrlich 实验结果 ${ }^{\left[{ }^{3}\right.}$.

通过以上分析可以看出,对于我们选取的计算方法和研究体系, 远离台阶处的第三个吸附 

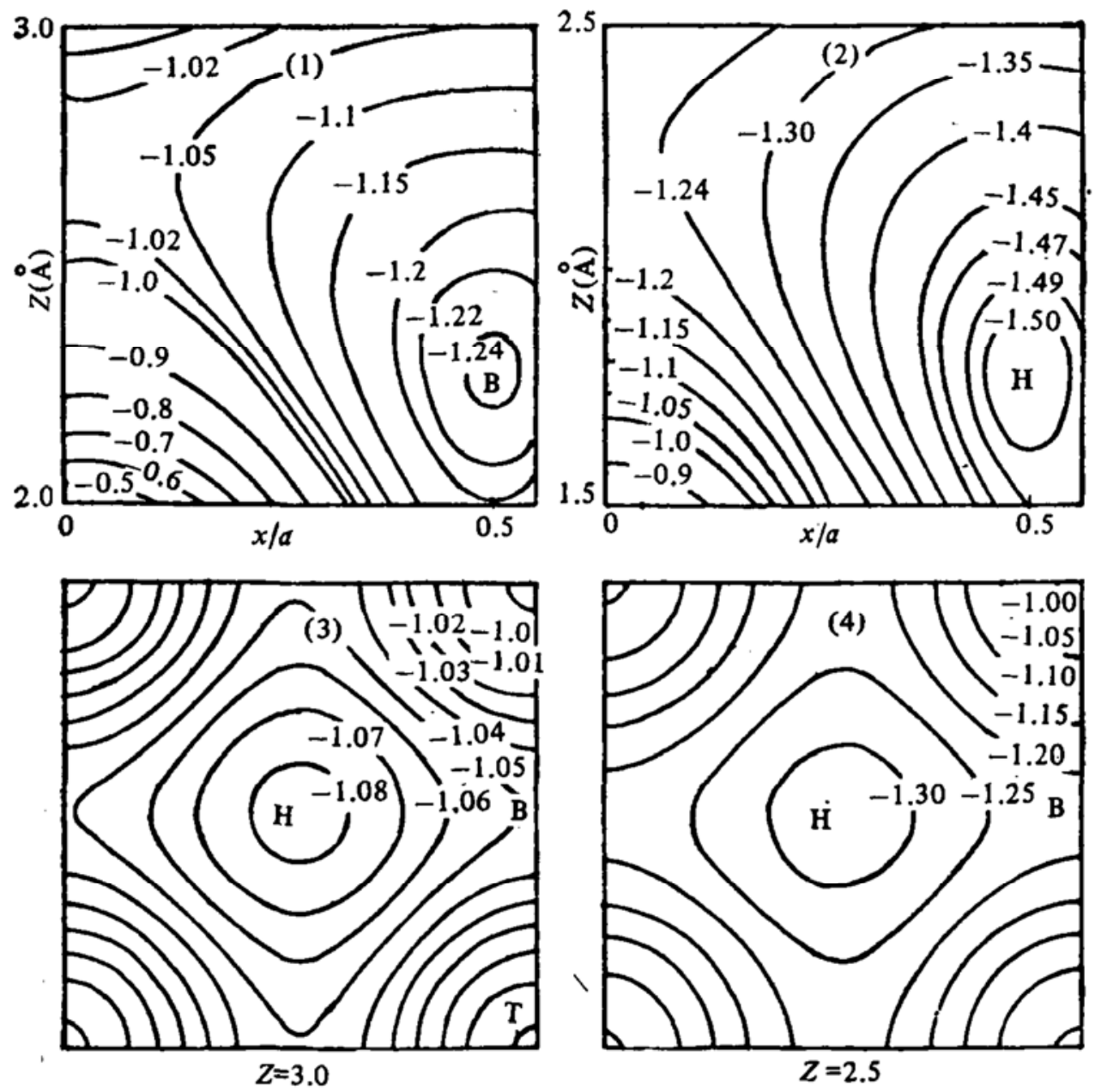

图 $1 \mathrm{Li}$ 原子在平坦表面吸附扩散势能面

能量单位为 $\mathrm{eV}$, 横坐标单位为晶格常数 $a$. (1)、(2) 分别为从顶位 $(\mathrm{T}) \rightarrow$ 桥位 (B) 和从桥位 $(B) \rightarrow$ 洞位 $(\mathrm{H})$ 的吸附扩散行为;(3)、(4) $\mathrm{Li}$ 距离表面的距离分别为 3.0 和 2.5 的平面扩散行为

表 3 上下台面的吸附参数

\begin{tabular}{|c|c|c|c|c|c|}
\hline & \multirow{2}{*}{ 位 置 } & & \multirow{2}{*}{ 结合能 $(\mathrm{eV})$} & \multicolumn{2}{|c|}{ 几何参数 } \\
\hline & & & & $x / a$ & 相对于台面的高侵 ( \\
\hline \multirow{4}{*}{$\begin{array}{l}\text { 上 } \\
\text { 台 } \\
\text { 面 }\end{array}$} & \multirow{2}{*}{ 吸附位 } & $\mathrm{H}_{1}$ & 1.50 & -1.50 & 1.80 \\
\hline & & $\mathrm{H}_{2}$ & 1.33 & -0.54 & 1.85 \\
\hline & \multirow{2}{*}{ 㜞 点 } & $s_{1}$ & 1.15 & -0.94 & 2.30 \\
\hline & & $S_{2}$ & 0.71 & 0.77 & 2.10 \\
\hline \multirow{5}{*}{$\begin{array}{l}\text { 下 } \\
\text { 台 } \\
\text { 面 }\end{array}$} & \multirow{3}{*}{ 吸附位 } & $\mathrm{H}_{3}$ & 2.18 & 0.50 & 1.60 \\
\hline & & $H_{\text {, }}$ & 1.62 & 1.50 & 1.75 \\
\hline & & $\mathrm{H}$, & 1.54 & 2.50 & 1.75 \\
\hline & \multirow{2}{*}{ 跤 点 } & $S_{3}$ & 1.56 & 1.20 & 2.10 \\
\hline & & s, & 1.28 & 2.0 & 2.30 \\
\hline
\end{tabular}

位,基本上具备平面吸附位的特性,这就是我们为消除边界效应所选取 Cluster 尺寸的依据。 

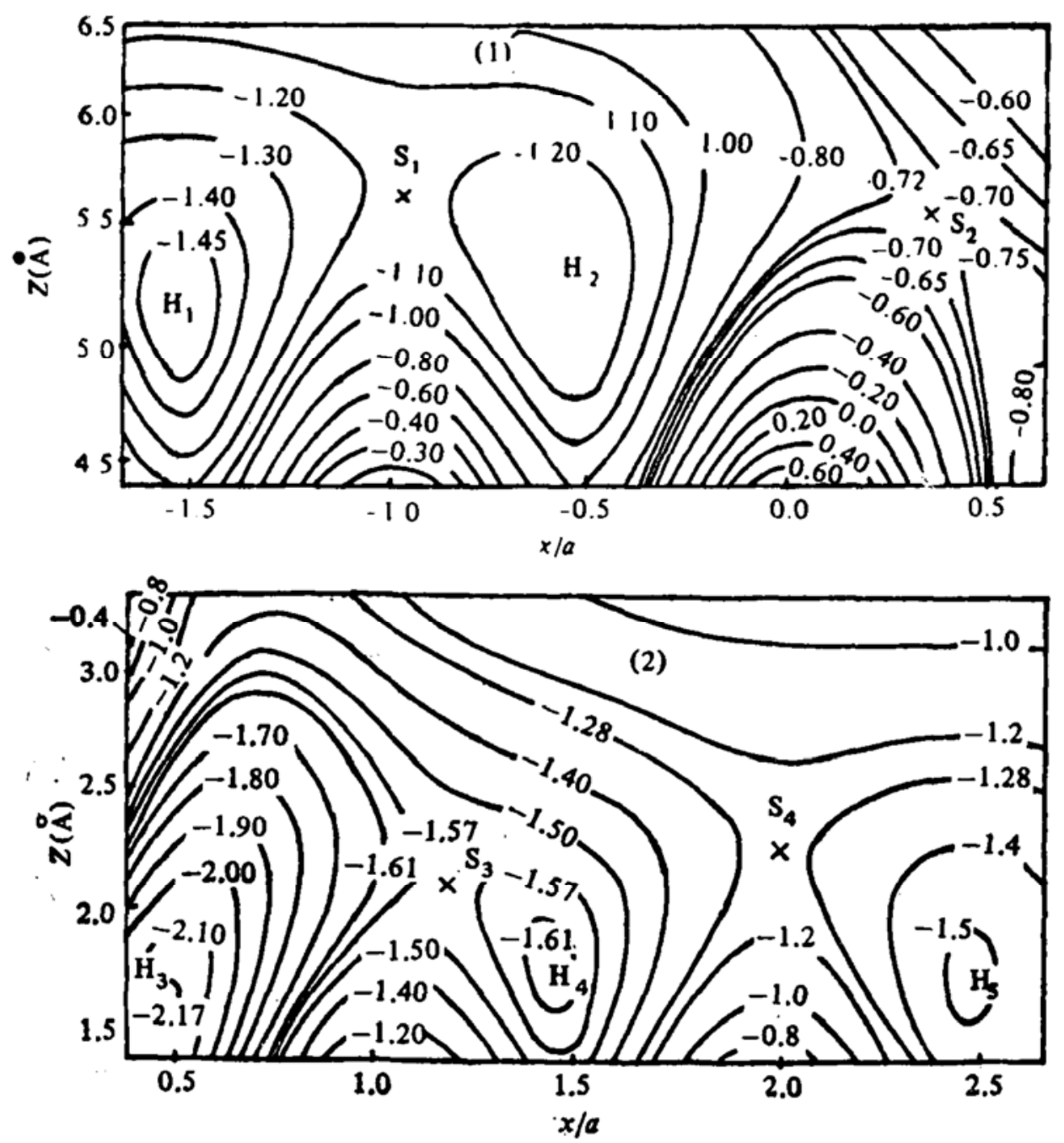

图 $2 \mathrm{Li}$ 原子在台阶缺陷表面吸附扩散势能面

级坐标 Z 为 $\mathrm{Li}$ 原子距下台面的高度, 横坐标 0 点为台阶位，(1) 上台面行为; (2) 下台面行为 表 4 上、下台面扩散活化能

\begin{tabular}{|c|c|c|}
\hline \multicolumn{2}{|c|}{ 扩 散 方 向 } & $E_{i} ;(\mathrm{eV})$ \\
\hline $\begin{array}{l}\text { 上 } \\
\text { 台 } \\
\text { 面 }\end{array}$ & $\begin{array}{l}\mathrm{H}_{1} \rightarrow \mathrm{S}_{1} \\
\mathrm{H}_{2} \rightarrow \mathrm{S}_{2} \\
\mathrm{H}_{2} \rightarrow \mathrm{S}_{1} \\
\mathrm{H}_{3} \rightarrow \mathrm{S}_{2}\end{array}$ & 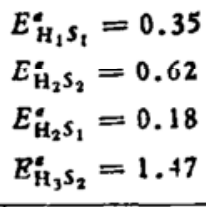 \\
\hline $\begin{array}{l}\text { 下 } \\
\text { 台 } \\
\text { 面 }\end{array}$ & $\begin{array}{l}\mathrm{H}_{3} \rightarrow \mathrm{S}_{3} \\
\mathrm{H}_{4} \rightarrow \mathrm{S}_{4} \\
\mathrm{H}_{3} \rightarrow \mathrm{S}_{4} \\
\mathrm{H}_{4} \rightarrow \mathrm{S}_{3}\end{array}$ & $\begin{array}{l}E_{\mathrm{H}_{4} S_{3}}=0.62 \\
E_{\mathrm{H}_{4} S_{4}}=0.34 \\
E_{\mathrm{H}_{4} s_{4}}=0.26 \\
E_{\mathrm{H}_{4} S_{3}}=0.06\end{array}$ \\
\hline 平面 & $\mathrm{H} \rightarrow \mathrm{S}$ & $E_{\mathrm{HS}}^{a}=0.30$ \\
\hline
\end{tabular}

\section{考文苦}

[1] Ander, L. W. et al., J. Chem. Phys., 10(1973), 5277.

[2] Johansson, P. K., Surf. Sci, 104(1981), 510.

[ 3 ] 王泽新等,化学学报, 48(1990), 11: 6 .

[ 4] Baetzold, R. C., Surf. Sci., 150(1985), 193.

[5] Huber, G. A. and Herzberg, G., Molecular Spectre and Molecular Strwture, IV, Reinhold, New York, 1179.

[6] Ehrlich, G., J. Chem. Phys., 44(1966), 1039, 1050. 